\title{
A $0535+26$ during the 2010 outburst observed with INTEGRAL and RXTE
}

\author{
Daniela Müller*† \\ Institut für Astronomie und Astrophysik, Universität Tübingen, Sand 1, D-72076 Tübingen, \\ Germany \\ E-mail: daniela.mueller@astro.uni-tuebingen.de
}

\section{Dmitry Klochkov}

Institut für Astronomie und Astrophysik, Universität Tübingen, Sand 1, D-72076 Tübingen

\author{
Isabel Caballero
}

CEA Saclay, France

\section{Rüdiger Staubert}

Institut für Astronomie und Astrophysik, Universität Tübingen, Sand 1, D-72076 Tübingen

\section{Andrea Santangelo}

Institut für Astronomie und Astrophysik, Universität Tübingen, Sand 1, D-72076 Tübingen

Many neutron star X-ray binary systems show high variability in the X-ray luminosity accompanied by spectral changes. For some systems, correlations between the source flux and the spectral continuum as well as the cyclotron line energy have been reported. We study the Be/X-ray binary system A 0535+26 during its 2010 outburst, which was monitored with INTEGRAL and RXTE. We analyze the phase-averaged, pulse-phase resolved and pulse-amplitude resolved spectra at different luminosity levels. The photon index decreases with increasing source flux, indicating a hardening of the spectrum during the decline of the outburst. The pulse-phase averaged cyclotron line energy is found to stay rather constant with changing flux. In the pulse-phase resolved analysis we observe indications for a spectral hardening and a positive cyclotron line energy-flux correlation for some phase bins, mainly during the secondary peak of the pulse profile. A similar behavior is seen in the pulse-amplitude resolved analysis, where a hardening of the spectrum with flux and an indication of a positive $E_{\text {cyc }}$-flux correlation is observed. It was proposed that the flux-related spectral changes reflect the accretion regime operating in the source. We discuss our results within the framework of different accretion regimes and compare our findings with the behavior observed in other sources (Her X-1, GX 304-1).

"An INTEGRAL view of the high-energy sky (the first 10 years)" 9th INTEGRAL Workshop and celebration of the 10th anniversary of the launch,

October 15-19, 2012

Bibliotheque Nationale de France, Paris, France

\footnotetext{
* Speaker.

${ }^{\dagger}$ This work has been partially funded by the DLR, grant 50 OR 1008, and by the Carl-Zeiss-Stiftung.
} 


\section{Introduction}

The Be/X-ray binary system A $0535+26$ was discovered in 1975 by the Ariel V satellites during a giant outburst [1]. The system shows a transient behavior undergoing normal and giant X-ray outbursts separated by quiescent phases [2]. The X-ray spectrum is traditionally modelled with a cutoff power law $[3,4]$ and shows absorption lines at around $50 \mathrm{keV}$ and $100 \mathrm{keV}[4,5]$ which are interpreted as fundamental and first harmonic cylcotron lines [4, 5]. For several accreting X-ray pulsars, a correlation between the cyclotron line energy and the source flux was reported. A negative correlation is seen in $\mathrm{V} 0332+53$ and $4 \mathrm{U} 0115+63[6,7,8,9]$ (for $4 \mathrm{U} 0115+63$ see, however, $[10,11])$, whereas a positive correlation is observed in Her X-1, GX 304-1 and Swift J1626.6-5156 $[12,13,14]$. This behavior is also present in the pulse-amplitude resolved analysis [15], which also reveals a softening of the X-ray continuum with flux in V 0332+53 and 4U 0115+63 and a hardening with flux in Her X-1 [15].

\section{Observations and spectral analysis}

We analyze INTEGRAL data (5 observations) and RXTE data ( 3 observations) of the April 2010 outburst of the source. The observations cover the maximum and the decay of the outburst. For our analysis, we use data from the PCA (5-60 keV) and HEXTE (20-145 keV) instruments of RXTE [16] and from JEM-X (5-30 keV) and ISGRI (20-145 keV) of INTEGRAL [17]. For the INTEGRAL data processing, we used OSA 9.0 and applied an additional gain correction for ISGRI data because of known problems in the energy calibration of OSA 9.0. The RXTE data were processed using HEASoft Version 6.11. We fitted the spectra with a power law with an exponential rolloff at higher energies, the cutoffpl model in XSPEC. We included a Gaussian emission line at around $6.4 \mathrm{keV}$. The residuals of the fit make the inclusion of an absorption line at around $\sim 44.4 \mathrm{keV}$ necessary. We modelled this line with a multiplicative absorption line with a Gaussian optical depth profile (gabs model in XSPEC). This line is usually interpreted as a cyclotron resonant scattering feature (CRSF). For the three RXTE observations, we also detect the first harmonic line at $\sim 104.2 \mathrm{keV}$. We analyze the dependence of the spectral parameters on source flux for the phase-averaged, pulse-phase resolved and pulse-amplitude resolved spectra.

\section{Results}

\subsection{Phase-averaged spectral analysis}

Figure 1 shows the dependence of the spectral parameters on source flux. The power law part of the spectral continuum gets harder with increasing flux as seen by a decrease of the photon index. The rolloff energy $E_{\text {rolloff }}$, however, shifts to smaller energies at higher fluxes. The data allow us to constrain $\Gamma$ and $E_{\text {rolloff }}$ simultaneously, which was checked with the $\chi^{2}$-contour plots. To find out whether the spectral continuum gets harder or softer with flux, that is to understand the

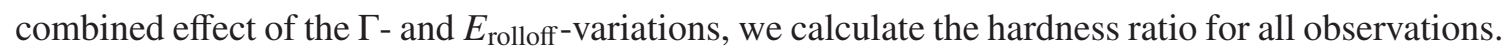
The hardness ratio shows, that the spectrum gets harder with increasing flux. The phase-averaged cyclotron line energy does not show any dependence on the flux during the outburst. This result is consistent with previous analyses of that source [e.g. 5, 18, 19]. 


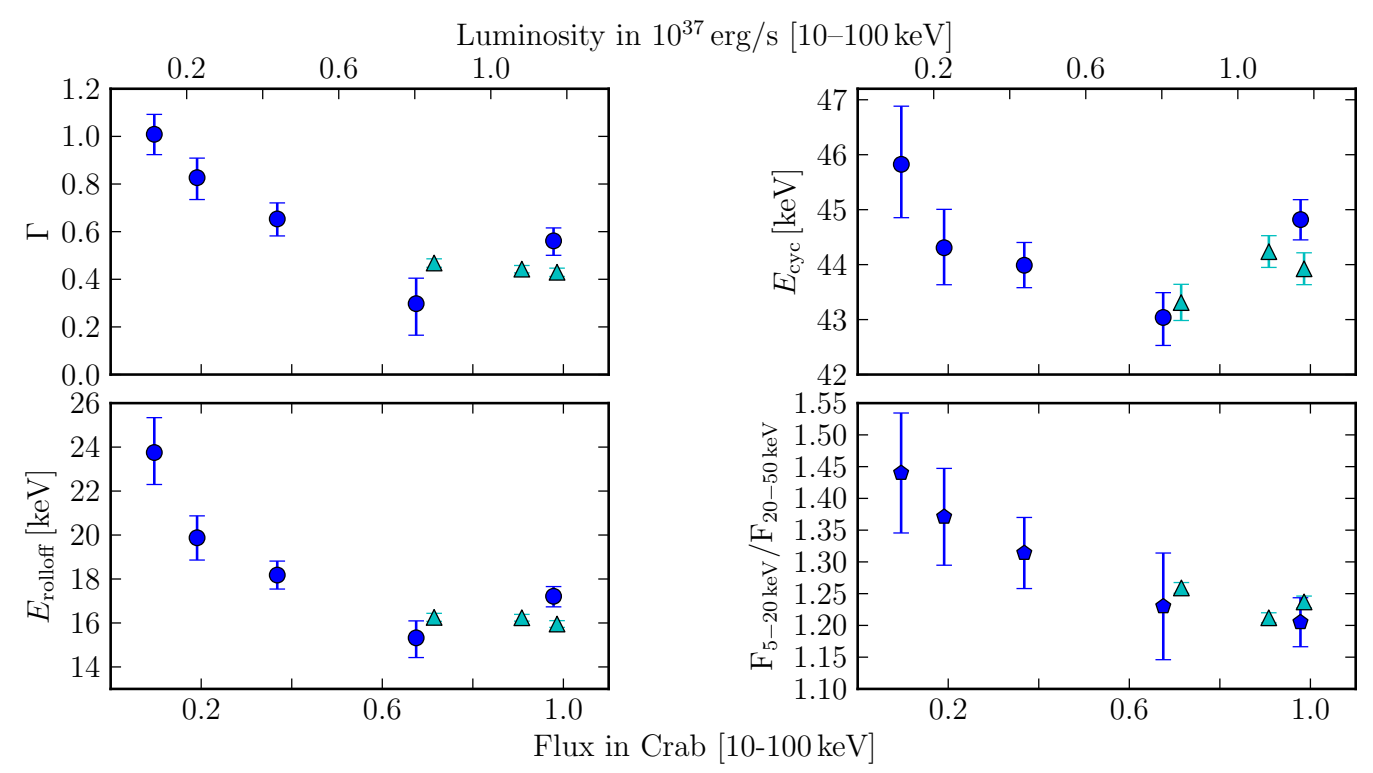

Figure 1: Fit parameters of the pulse-averaged spectra. INTEGRAL observations are marked with circles, RTXE observations with triangles. We plot the photon index $\Gamma$, the rolloff energy $E_{\text {rolloff }}$, the cyclotron line energy $E_{\text {cyc }}$ and the hardness ratio as a function of source flux and luminosity. The errors are $1 \sigma$.

\subsection{Pulse-phase resolved spectral analysis}

We also perform a spectral analysis for the two peaks of the pulse profile (see Fig. 2). We extracted spectra for all RXTE and INTEGRAL observations for the main peak (phase interval 0.1-0.5) and for the secondary peak (phase interval 0.6-1.0) (see Figure 2). In Figure 2 we plot the corresponding spectral parameters for the photon index $\Gamma$ and the cyclotron line energy $E_{\text {cyc }}$ as a function of the source flux/luminosity. For the middle plot we fix $E_{\text {rolloff }}$ in order to characterize the hardness of the continuum by the photon index alone. We find that the main peak shows a slightly harder spectrum (lower photon index) compared to the secondary peak. The spectrum of the secondary peak gets harder with increasing source flux (green points in Fig. 2), whereas for the main peak $\Gamma$ stays constant (pink points). For the main peak, the cyclotron line energy $E_{\text {cyc }}$ does not change significantly, whereas it increases with flux for the secondary peak.

\subsection{Pulse-amplitude resolved analysis}

Since A $0535+26$ shows high pulse-to-pulse variability, we also analyzed the spectra selecting pulse amplitudes, similarly to [15]. To compare our results with [15], we chose the secondary peak of the pulse profile, for which we calculated the amplitudes of individual pulses in the light curve. We then grouped together pulses in different pulse amplitude intervals and extracted spectra. Here, we report the results for the RXTE observations. In order to increase the statistics, we grouped together all three observations (which were performed at similar luminosity levels). To characterize the continuum by the photon index alone, we fixed the rolloff energy to $E_{\text {rolloff }}=15.2 \mathrm{keV}$. As shown in Figure 3, the photon index $\Gamma$ decreases with increasing pulse height amplitude, meaning a hardening of the spectrum with pulse height. The cyclotron line energy $E_{\text {cyc }}$ shows a positive correlation with pulse height. Our results are, thus, consistent with the behavior described in [15]. 

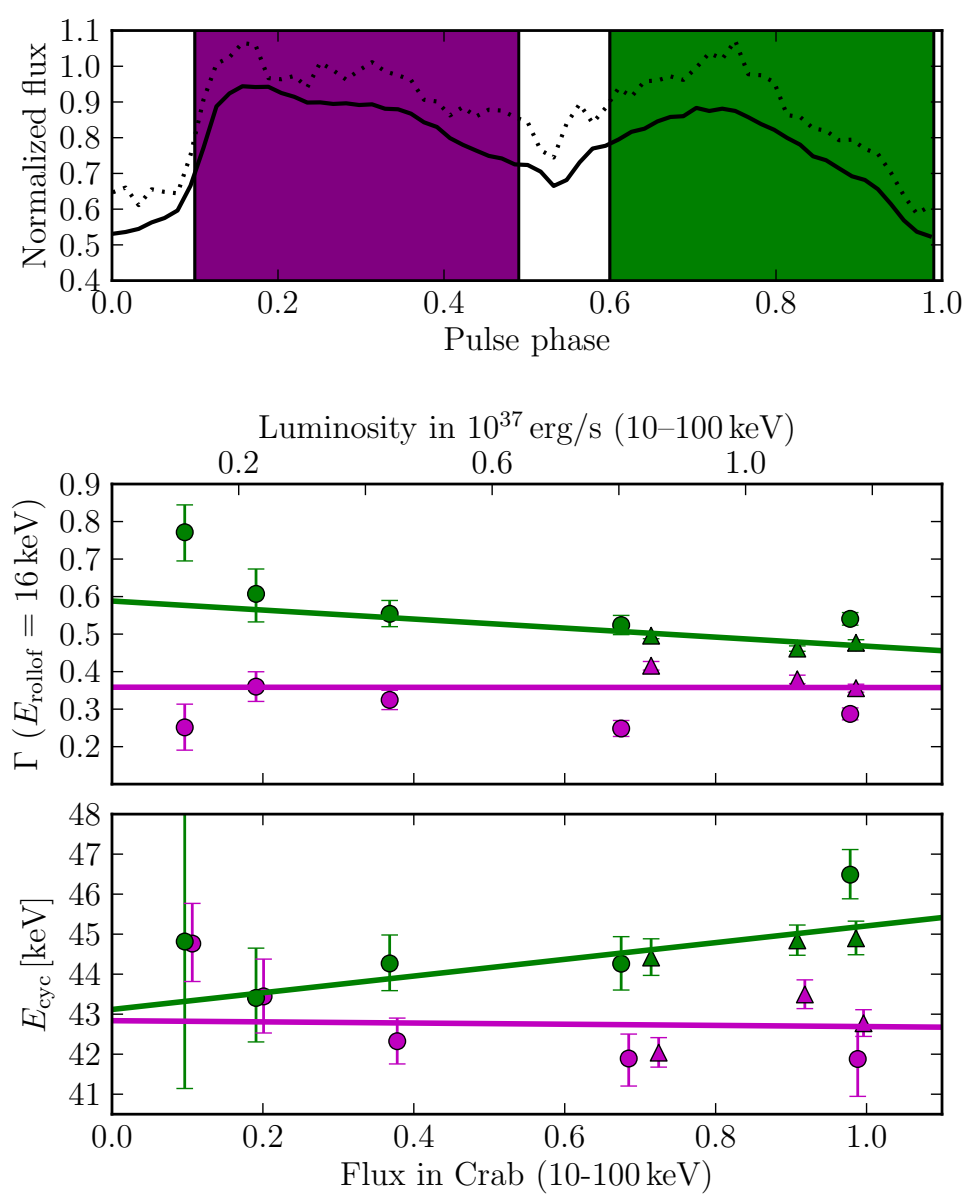

Figure 2: Upper plot. The averaged 3-80 keV INTEGRAL pulse profile (continuous line) and 3-60 keV RXTE pulse profile (dotted line). We mark the phase intervals which we selected for the pulse-phaseresolved analysis with shaded areas in pink (main peak, phase interval 0.1-0.5) and green (secondary peak, phase interval 0.6-1.0). Lower plot. The parameters of the photon index $\Gamma$ for fixed $E_{\text {rolloff }}=16.0 \mathrm{keV}$ and the cyclotron line energy $E_{\text {cyc. }}$. We plot the fit values for the main peak (pink) and for the secondary peak (green). INTEGRAL observations are, again, marked with circles, RXTE observations with triangles. The errors are $1 \sigma$.

\section{Conclusions}

As discussed in $[15,20]$, there appear to exist two distinct groups of sources which differ in the flux-dependence of the spectral hardness and of the cyclotron line energy. [20] report the values of the critical luminosity, which separates two accretion regimes. For A 0535+26, they estimated a value of $L_{\text {crit }} \sim 6.78 \times 10^{37} \mathrm{erg} / \mathrm{s}$. We observed A $0535+26$ during the 2010 outburst at a peak luminosity of about $1.1 \times 10^{37} \mathrm{erg} / \mathrm{s}$ (assuming a distance of $2 \mathrm{kpc}$ ). We analyzed the phaseaveraged, the pulse-phase resolved and the pulse-amplitude resolved spectra at different luminosity levels of this outburst. We observe a hardening of the photon index with increasing luminosity. But unlike other sources with sub-critical luminosities (Her X-1, GX 304-1), we do not see a positive correlation for the phase-averaged cyclotron line energy with source flux. However, an indication of 


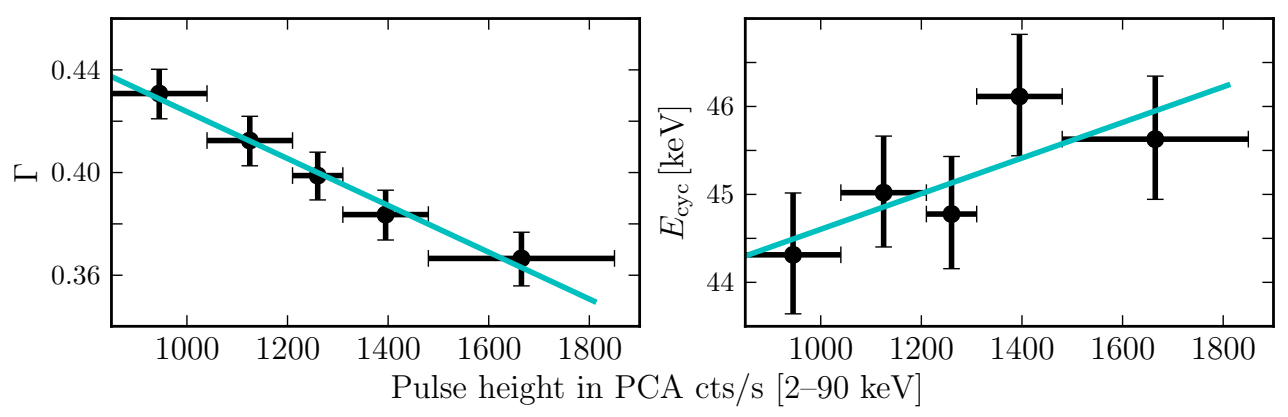

Figure 3: Fit values for the RXTE pulse-amplitude resolved analysis. The photon index $\Gamma$ and the cyclotron line energy $E_{\mathrm{cyc}}$ are shown as function of pulse height. For the left plot, we fixed the rolloff energy to $E_{\text {rolloff }}=15.2 \mathrm{keV}$ to characterize the hardness of the spectral continuum by one parameter alone. For the plot on the right all parameters are kept free. The errors are $1 \sigma$.

a positive $E_{\mathrm{cyc}}$-flux correlation is seen in a certain pulse-phase interval (accompanied by a spectral hardening with flux) and in the pulse-amplitude resolved analysis. This supports the idea that A $0535+26$ operates in a sub-critical accretion regime during the 2010 outburst.

\section{References}

[1] Rosenberg, F. D., Eyles, C. J., Skinner, G. K., et al., 1975, Nature, 256, 628-630

[2] Motch, C., Stella, L., Janot-Pacheco, E., \& Mouchet, M., ApJ, 369 (1991), 490

[3] Ricketts, M. J., Turner, M. J. L., Page, C. G., \& Pounds, K. A., 1975, Nature, 256, 631-633

[4] Kendziorra, E., Kretschmar, P., Pan, H. C., et al., A\&A, 291, L31-L34

[5] Caballero, I., Kretschmar, P., Santangelo, A., et al., 2007, A\&A, 465, L21-L24

[6] Tsygankov, S. S., Lutovinov, A. A., Churazov, E. M., \& Sunyaev, R. A., 2006, MNRAS, 371, $19-28$

[7] Tsygankov, S. S., Lutovinov, A. A., Churazov, E. M., \& Sunyaev, R. A., 2007, Astronomy Letters, 33, 368-384

[8] Tsygankov, S. S., Lutovinov, A. A., \& Serber, A. V. , 2010, MNRAS, 401, 1628-1635

[9] Mowlavi, N., Kreykenbohm, I., Shaw, S. E., et al., 2006, A\&A, 451, 187-194

[10] Müller, S., Ferrigno, C., Kühnel, M., et al., 2012, astro-ph.HE 1211.6298

[11] Müller, S. et al., 2013, PoS (INTEGRAL12), this volume

[12] Staubert, R., Shakura, N. I., Postnov, K., et al., 2007, A\&A, 465, L25-L28

[13] Klochkov, D., Doroshenko, V., Santangelo, A., et al., 2012, A\&A, 542, L28 
[14] DeCesar, M. E., Boyd, P. T., Pottschmidt, K., et al., 2012, ApJ, 762, 61

[15] Klochkov,D., Staubert, R., Santangelo, A., et al., 2011, A\&A, 532, A126

[16] Bradt, H. V., Rothschild, R. E., \& Swank, J. H., 1993, A\&AS, 97, 355-360

[17] Winkler, C., Courvoisier, T. J.-L., Di Cocco, G., et al., 2003, A\&A, 411, L1-L6

[18] Caballero, I., Santangelo, A., Kretschmar, P., et al., 2008, A\&A, 480, L17-L20

[19] Caballero, I., Pottschmidt, K., Santangelo, A., et al., 2011, astro-ph.HE 1107.3417

[20] Becker, P. A., Klochkov, D., Schönherr, G., et al., 2012, A\&A, 544, A123

[21] Ferrigno, C., Falanga, M., Bozzo, E., et al., 2011, 532, A76 